\title{
Congenital Central Hypoventilation Syndrome: Optimizing Care with a Multidisciplinary Approach
}

\author{
Ajay S Kasi $\mathbb{D}^{\prime}$, Hong $\mathrm{Li}^{2}$, Kelli-Lee Harford', Humphrey V Lam ${ }^{3}$, Chad Mao ${ }^{4}$, April M Landry ${ }^{5}$, \\ Sarah G Mitchell $\mathbb{D}^{6}{ }^{\text {, Matthew S Clifton }}{ }^{7}$, Roberta M Leu' \\ 'Department of Pediatrics, Division of Pediatric Pulmonology and Sleep Medicine, Emory University, Children's Healthcare of Atlanta, Atlanta, GA, \\ USA; ${ }^{2}$ Department of Human Genetics, Emory University, Children's Healthcare of Atlanta, Atlanta, GA, USA; ${ }^{3}$ Division of Pediatric Anesthesiology, \\ Emory University, Children's Healthcare of Atlanta, Atlanta, GA, USA; ${ }^{4}$ Department of Pediatrics, Division of Pediatric Cardiology, Emory University, \\ Children's Healthcare of Atlanta, Atlanta, GA, USA; ${ }^{5}$ Division of Pediatric Otorhinolaryngology, Emory University, Children's Healthcare of Atlanta, \\ Atlanta, GA, USA; ${ }^{6}$ Department of Pediatrics, Division of Pediatric Hematology and Oncology, Emory University, Children's Healthcare of Atlanta, \\ Atlanta, GA, USA; ${ }^{7}$ Division of Pediatric Surgery, Emory University, Children's Healthcare of Atlanta, Atlanta, GA, USA
}

Correspondence: Ajay S Kasi, Department of Pediatrics, Division of Pediatric Pulmonology and Sleep Medicine, Emory University, Children's Healthcare of Atlanta, I 400 Tullie Road NE, Atlanta, GA, 30329, USA, Tel +I 404785 5437, Fax +I 404785 9087, Email ajay.kasi@emory.edu

\begin{abstract}
Congenital central hypoventilation syndrome (CCHS) is a rare genetic disorder affecting respiratory control and autonomic nervous system function caused by variants in the paired-like homeobox $2 \mathrm{~B}(\mathrm{PHOX} 2 \mathrm{~B})$ gene. Although most patients are diagnosed in the newborn period, an increasing number of patients are presenting later in childhood, adolescence, and adulthood. Despite hypoxemia and hypercapnia, patients do not manifest clinical features of respiratory distress during sleep and wakefulness. CCHS is a lifelong disorder. Patients require assisted ventilation throughout their life delivered by positive pressure ventilation via tracheostomy, noninvasive positive pressure ventilation, and/or diaphragm pacing. At different ages, patients may prefer to change their modality of assisted ventilation. This requires an individualized and coordinated multidisciplinary approach. Additional clinical features of CCHS that may present at different ages and require periodic evaluations or interventions include Hirschsprung's disease, gastrointestinal dysmotility, neural crest tumors, cardiac arrhythmias, and neurodevelopmental delays. Despite an established $P H O X 2 B$ genotype and phenotype correlation, patients have variable and heterogeneous clinical manifestations requiring the formulation of an individualized plan of care based on collaboration between the pulmonologist, otolaryngologist, cardiologist, anesthesiologist, gastroenterologist, sleep medicine physician, geneticist, surgeon, oncologist, and respiratory therapist. A comprehensive multidisciplinary approach may optimize care and improve patient outcomes. With advances in CCHS management strategies, there is prolongation of survival necessitating high-quality multidisciplinary care for adults with CCHS.
\end{abstract}

Keywords: congenital central hypoventilation syndrome, multidisciplinary care, diaphragm pacing, tracheostomy, noninvasive ventilation, CCHS

\section{Introduction}

Congenital central hypoventilation syndrome (CCHS) is a rare genetic disorder affecting control of breathing and autonomic nervous system (ANS) function caused by variants in the paired-like homeobox 2B (PHOX2B) gene. ${ }^{1}$ Mellins et al reported the first case of CCHS in an infant with alveolar hypoventilation of central nervous origin characterized by severe chronic respiratory acidosis alleviated by assisted ventilation (AV) after excluding primary cardiac, pulmonary, neuromuscular, and chest wall disease. Despite AV with a negative pressure ventilator, the infant developed pulmonary hypertension and died at 14 months of age due to complications from congestive heart failure. ${ }^{2}$ Since the initial report of CCHS in 1970, over 1000 cases have been reported worldwide. ${ }^{1}$ The incidence of CCHS is estimated to be one in 148,000-200,000 live births. ${ }^{3}$ Patients with CCHS generally present in the neonatal period with respiratory failure, apnea, cyanosis, hypoxemia, and hypoventilation that are more severe during sleep than during wakefulness. Some individuals with CCHS may present later in infancy, childhood, and even during adulthood with respiratory failure following respiratory infections or general anesthesia. ${ }^{1,45}$ Affected patients do not manifest signs of 
respiratory distress such as tachypnea, nasal flaring, and chest retractions despite hypoxemia and hypercapnia making the diagnosis and management challenging. ${ }^{1,3,6}$

The identification of the PHOX2B gene as the disease-causing link to CCHS has led to earlier confirmation of the diagnosis, institution of $\mathrm{AV}$, and evaluation for associated conditions. ${ }^{1,7}$ With advances in management strategies, increased awareness of CCHS, and multidisciplinary care, children with CCHS are surviving into adulthood with a good quality of life. ${ }^{8,9}$ In this review, we will discuss the clinical features of CCHS and optimizing management using a multidisciplinary care approach.

\section{Pathophysiology}

In CCHS, there is impaired central regulation of respiration that is worse during sleep than wakefulness. Decreased tidal volumes and monotonous respiratory rates in patients with CCHS leads to alveolar hypoventilation and hypoxemia that is worse during nonrapid eye movement (NREM) sleep than during rapid eye movement (REM) sleep. ${ }^{1,10}$ Patients with CCHS have absent or decreased ventilatory responses to hypoxemia and hypercapnia during both sleep and wakefulness. In addition, patients may have abnormal arousal responses to hypercapnia during sleep. ${ }^{10,11}$ The respiratory control abnormality in CCHS is predicted to be in the central integration of peripheral and central chemoreceptor signals. ${ }^{12,13}$

Efforts to further understand the impaired chemoreceptor function in CCHS have examined their heart rate and ventilatory responses to exercise. Using an incremental walking treadmill protocol in CCHS children requiring continuous AV, Silvestri et al reported hypoxemia, hypercapnia, and lower increase in heart rate compared to healthy controls. ${ }^{14}$ Incremental treadmill exercise testing in CCHS children requiring AV during sleep demonstrated mild gas exchange abnormalities at maximum exercise. Compared to healthy controls, Paton et al demonstrated that minute ventilation was lower in CCHS children with mild increase in tidal volumes and greater increase in respiratory frequency. They speculated that the increase in respiratory rate during exercise was stimulated by limb movement. ${ }^{15}$ Gozal et al later demonstrated that passive movement of the lower limbs during sleep and wakefulness increased alveolar ventilation in CCHS children. These findings were attributed to the potential activation of mechanoreceptors that stimulated respiration in CCHS. ${ }^{16,17}$ Interestingly, in children with CCHS, activities requiring mental concentration such as solving arithmetic problems, reading, and playing video games did not lead to hypoventilation. ${ }^{18}$ These studies demonstrate that physical activity can affect ventilation in patients with CCHS.

\section{Genetics}

CCHS is caused by monoallelic pathogenic variants in the $P H O X 2 B$ gene that encodes a transcription factor, the pairedlike homeobox $2 \mathrm{~B}$ protein, that plays a role in the early development of the ANS. The PHOX2B gene is located in chromosome 4p12 and has 3 exons that code for 314 amino acids. ${ }^{1}$ The largest exon 3 normally contains a repeat sequence code for 20 alanines. There are 2 types of mutations in PHOX2B causing CCHS: polyalanine repeat mutations (PARMs) and nonpolyalanine repeat mutations (NPARMs). PARMs refer to expansion in the polyalanine repeat region in exon 3 and account for $90 \%$ of CCHS cases. In patients with PARMs, the normal 20-alanine tract is expanded to 24-33 alanine repeats with resultant genotypes of 20/24 - 20/33. The most common genotypes in CCHS are 20/25, 20/26, and 20/27 PARMs. NPARMs account for $10 \%$ of CCHS patients, including missense, nonsense, frameshift, splice site, and stop codon variants. Most of the NPARMs are in exon 2 and 3 of PHOX2B. Partial or whole gene deletions of PHOX2B account for $<1 \%$ cases and are associated with variable phenotypes. ${ }^{1,9}$

Generally, there is a correlation between the PHOX2B genotype and the phenotypic features of alveolar hypoventilation, Hirschsprung's disease (HD), cardiac arrhythmias, ANS dysregulation, and neural crest tumors (NCT). Relatively less severe clinical features may be seen in patients with shorter PARMs such as 20/25 and 20/26 requiring AV only during sleep and without associated HD and NCT. Patients with genotypes 20/27 - 20/33 may require full-time AV and have associated HD. NPARMs may be associated with more severe phenotypes including need for continuous AV, HD, and increased NCT risk. ${ }^{1}$ However, there can be clinical variability with late-onset CCHS in some patients with 20/24 and 20/25 PARMs and NPARMs. Both intrafamilial and interfamilial variable expression and incomplete penetrance among individuals with $P H O X 2 B$ variants have been reported, indicating other genetic modifiers and/or environmental factors are contributory to the broad spectrum of clinical manifestations. ${ }^{7,19,20}$ 


\section{Clinical Features}

Patients with CCHS generally present in the newborn period with apnea, cyanosis, hypoxemia, hypoventilation, and/or persistent respiratory failure with inability to wean off $\mathrm{AV} .{ }^{1,3}$ Alveolar hypoventilation is the characteristic clinical feature in CCHS. Hypoxemia, hypoventilation, and central apneas are worse during NREM sleep but may be present during REM sleep and wakefulness. ${ }^{1,10}$ Due to the lack of subjective and objective responses to hypoxemia and hypercapnia, patients with CCHS do not manifest the typical clinical signs of respiratory compromise such as nasal flaring, tachypnea, and chest retractions. ${ }^{6}$ Despite progressive hypercapnia and hypoxemia, infants with CCHS do not increase their respiratory effort or frequency and may not arouse or appear distressed. The discordance between the clinical presentation and severity of gas exchange abnormalities contributes to the diagnostic and management challenges in CCHS. Beyond the newborn period, infants with CCHS can present with brief resolved unexplained events (BRUE), unexplained apnea, or pulmonary hypertension due to hypoxemia. Patients with less severe clinical features of CCHS may present later in childhood, adolescence, and even during adulthood with unexplained persistent respiratory failure, seizures, or pulmonary hypertension following respiratory infections or general anesthesia., ${ }^{5,19}$

CCHS is associated with ANS dysregulation that encompasses HD, NCT, cardiovascular disturbances, reduced basal body temperature, pupillary abnormalities, abnormal perception of dyspnea and anxiety, and breath-holding spells. ${ }^{1,21-23}$ Cardiovascular abnormalities include bradycardia, decreased heart rate variability, transient asystole, blood pressure abnormalities, and life-threatening cardiac arrhythmias requiring implantation of a cardiac pacemaker. ${ }^{22,24}$ Patients with CCHS and gastrointestinal motility disorders may present with constipation and esophageal dysmotility. Although HD is more prevalent in patients with NPARMs (80\%) compared to those with PARMs (19\%), it was recently reported even in patients with the relatively milder 20/25 PARM genotype, suggesting that patients with constipation should be assessed for HD regardless of their PHOX2B genotype. ${ }^{1,23}$ NCTs such as neuroblastoma, ganglioneuroblastoma, and ganglioneuroma are much more prevalent in patients with NPARM compared to those with PARM. ${ }^{1}$ Neurodevelopmental delays can occur as early as in preschool children potentially due to $P H O X 2 B$ gene dysfunction and/or recurrent hypoxemia and hypoventilation. ${ }^{1,25}$

When the diagnosis of CCHS is suspected, a diagnostic algorithm may aid in establishing the diagnosis. ${ }^{3}$ Alveolar hypoventilation should be confirmed by polysomnography with capnography and/or serial blood gas evaluations with elevated partial pressure of carbon dioxide $\left(\mathrm{P}_{\mathrm{CO} 2}\right)$. Polysomnographic abnormalities in CCHS include hypoventilation and hypoxemia that is more severe in NREM sleep than REM sleep, abnormal arousal and heart rate responses to hypoxemia, and central apneas. ${ }^{1,10}$ PHOX2B genetic studies should be performed to confirm the diagnosis of CCHS. While awaiting the results of the genetic tests, the patient should be evaluated for other etiologies of hypoventilation with a multidisciplinary approach in collaboration with a pediatric pulmonologist, neurologist, and geneticist. These evaluations include chest radiography, airway endoscopy, electrocardiogram and echocardiogram, magnetic resonance imaging (MRI) of the brain and brainstem, diaphragm ultrasound or fluoroscopy, metabolic studies, and comprehensive neurological evaluation. ${ }^{1,3}$

All the clinical features of CCHS do not present at birth or during the initial diagnosis of CCHS. Some disease manifestations can present at different ages requiring serial evaluations. Therefore, close monitoring of each patient and regular surveillance studies are essential to identify early manifestations and to formulate an individualized plan of care. ${ }^{7}$ These evaluations include comprehensive respiratory assessments during sleep and wakefulness to assess ventilatory needs, polysomnography with titration of ventilator settings to maintain oxygen saturation $\left(\mathrm{SpO}_{2}\right) \geq 95 \%$ and end-tidal carbon dioxide $\left(\mathrm{ETCO}_{2}\right)$ between $35-40 \mathrm{~mm} \mathrm{Hg}$, echocardiogram to assess for pulmonary hypertension, ambulatory cardiac monitoring to detect cardiac arrhythmias, imaging and urine studies for NCT, ophthalmologic and neurocognitive testing, and airway endoscopy for patients with a tracheostomy (Table 1). ${ }^{1,3}$ Coordinating these tests during in-hospital evaluations may be beneficial in formulating a comprehensive management plan.

\section{Optimizing Care with a Multidisciplinary Approach}

CCHS is not just a disease of the respiratory system. Many organ systems are affected. Each area of care interacts with another and influences the overall health of the patient. In addition, the clinical features of CCHS can evolve over time. ${ }^{7}$ 
Table I Recommended Evaluations in Patients with CCHS

\begin{tabular}{|c|c|c|c|}
\hline & Evaluation & Frequency & $\begin{array}{l}\text { PHOX2B } \\
\text { Genotype }\end{array}$ \\
\hline Pulmonary & $\begin{array}{l}\text { Comprehensive physiologic testing during sleep and wakefulness } \\
\text { including continuous pulse oximetry, cardiorespiratory monitoring, } \\
\text { capnography, and polysomnography }\end{array}$ & $\begin{array}{l}<3 \text { years of age: every } 6 \\
\text { months } \\
\geq 3 \text { years: annually }\end{array}$ & $\begin{array}{l}\text { All PHOX2B PARM } \\
\text { and NPARM }\end{array}$ \\
\hline Cardiovascular & $\begin{array}{l}\text { Ambulatory cardiac monitoring ( } \geq 72 \text { hours), blood pressure, and } \\
\text { echocardiogram }\end{array}$ & Annually & $\begin{array}{l}\text { All PHOX2B PARM } \\
\text { and NPARM }\end{array}$ \\
\hline Gastrointestinal & $\begin{array}{l}\text { Barium enema or anorectal manometry. Confirmation by rectal } \\
\text { biopsy. }\end{array}$ & $\begin{array}{l}\text { At initial diagnosis and } \\
\text { subsequently if symptoms } \\
\text { appear }\end{array}$ & $\begin{array}{l}20 / 26-20 / 33 \\
\text { PARM and NPARM }\end{array}$ \\
\hline Neurodevelopmental & Comprehensive neurocognitive tests & $\begin{array}{l}<3 \text { years of age: every } 6 \\
\text { months } \\
\geq 3 \text { years: annually }\end{array}$ & $\begin{array}{l}\text { All PHOX2B PARM } \\
\text { and NPARM }\end{array}$ \\
\hline Oncologic & $\begin{array}{l}\text { Chest radiograph, abdominal ultrasound, and urine catecholamines } \\
\text { (homovanillic acid and vanillylmandelic acid). }\end{array}$ & $\begin{array}{l}0-6 \text { years of age: every } 3 \\
\text { months } \\
6-10 \text { years of age: every } 6 \\
\text { months } \\
>10 \text { years of age: per } \\
\text { oncologist recommendation }\end{array}$ & $\begin{array}{l}20 / 28-20 / 33 \\
\text { PARM and NPARM }\end{array}$ \\
\hline Ophthalmologic & Comprehensive ocular testing by an ophthalmologist & Annually & $\begin{array}{l}\text { All PHOX2B PARM } \\
\text { and NPARM }\end{array}$ \\
\hline
\end{tabular}

Note: Data from these studies. ${ }^{1,3,41 .}$

Abbreviations: NPARM, nonpolyalanine repeat expansion mutation; PARM, polyalanine repeat expansion mutation; PHOX2B, paired-like homeobox 2B.

Therefore, a coordinated comprehensive multidisciplinary approach to care is vital to ensure the best possible outcomes throughout the lifespan. Coordination of care among disciplines can be complicated and challenging for the patient and the family (Figure 1). Therefore, a primary point of contact to coordinate and review patient care from a holistic, multidisciplinary perspective is critical. In CCHS, the pediatric pulmonologist generally takes the lead in care coordination and facilitates communication among subspecialists. A primary point of contact for the patients and families in the pediatric pulmonology office is typically a nurse, nurse case manager, or a nurse care coordinator whose role is to provide information, education, insurance navigation and prior authorization support, facilitate appointment scheduling, coordination with different specialists, and reduce burden on the patient-family unit. At our institution, the CCHS multidisciplinary team is comprised of a pediatric pulmonologist, sleep medicine physician, sleep psychologist, otolaryngologist, cardiologist, anesthesiologist, geneticist, gastroenterologist, pediatric surgeon, oncologist, respiratory therapist, social worker, and nurse case manager (Figure 2). ${ }^{26}$ The goal of comprehensive multidisciplinary care in CCHS is to provide consistent and coordinated care that aligns with current CCHS care guidelines, improves quality of life, and optimizes outcome. ${ }^{1,3}$

\section{Respiratory Management}

Respiratory abnormalities in CCHS falls into a spectrum, ranging from central sleep apnea, sleep-disordered breathing, hypoventilation during sleep requiring $\mathrm{AV}$, to hypoventilation during wakefulness and sleep that requires continuous AV. ${ }^{1,6}$ Ventilatory support is life support for patients with CCHS. CCHS is a lifelong disorder, and patients require AV throughout their life. The AV requirement may range from sleep-only to full-time ventilator dependence and generally correlates with the $P H O X 2 B$ genotype. ${ }^{1,6}$ Currently no respiratory stimulant medications have demonstrated effectiveness in obviating the requirement of $A{ }^{3}{ }^{3}$ Supplemental oxygen therapy alone may improve oxygenation but is insufficient to alleviate hypoventilation. Therefore, AV should be provided either by positive pressure ventilation via tracheostomy 


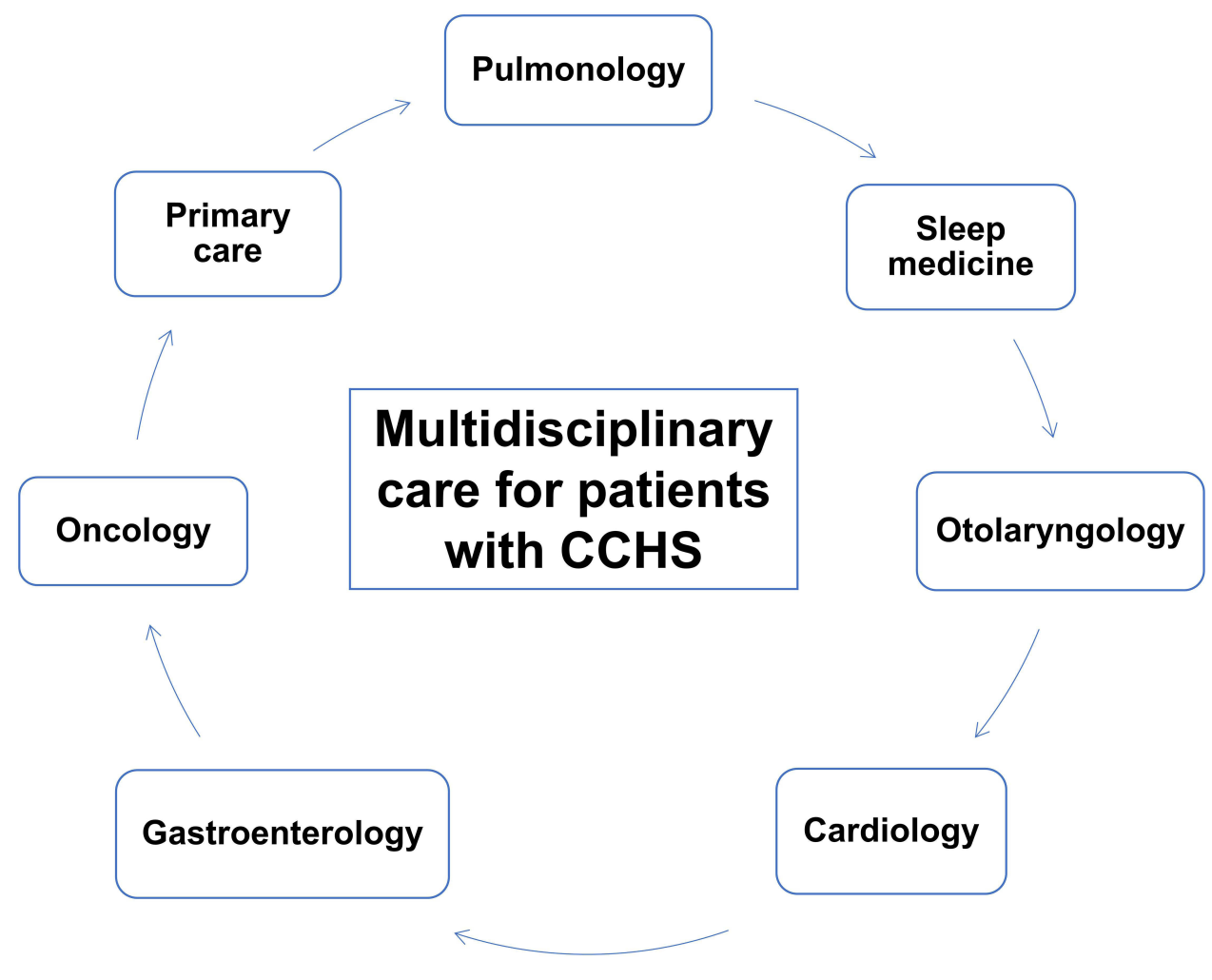

Figure I Multidisciplinary care for patients with congenital central hypoventilation syndrome.

Abbreviation: $\mathrm{CCHS}$, congenital central hypoventilation syndrome.

\begin{tabular}{|c|c|}
\hline $\begin{array}{l}\text { Pediatric Intensivist/ } \\
\text { Neonatologist }\end{array}$ & $\begin{array}{l}\text { - Management of respiratory failure at presentation } \\
\text { - Diagnostic evaluations for } \mathrm{CCHS}\end{array}$ \\
\hline Pediatric pulmonologist & $\begin{array}{l}\text { - Management of assisted ventilation } \\
\text { - Coordination of care }\end{array}$ \\
\hline Sleep medicine physician & $\begin{array}{l}\text { - Polysomnography with titration of assisted ventilation } \\
\text { - Optimizing use of NPPV }\end{array}$ \\
\hline Pediatric otolaryngologist & $\begin{array}{l}\text { - Tracheostomy management } \\
\text { - Endoscopic airway evaluation }\end{array}$ \\
\hline Pediatric cardiologist & $\begin{array}{l}\text { - Surveillance echocardiogram, ambulatory cardiac monitoring } \\
\text { - Cardiac pacemaker management }\end{array}$ \\
\hline Pediatric oncologist & - Surveillance and management of neural crest tumors \\
\hline Pediatric genetics & $\begin{array}{l}\text { - Genetic counseling } \\
\text { - Parental } P H O X 2 B \text { genetic tests }\end{array}$ \\
\hline Pediatric surgeon & $\begin{array}{l}\text { - Thoracoscopic placement of phrenic nerve pacers for DP } \\
\text { - Surgical management of neural crest tumors }\end{array}$ \\
\hline $\begin{array}{c}\text { Pediatric } \\
\text { gastroenterologist }\end{array}$ & $\begin{array}{l}\text { - Evaluation and management of Hirschsprung's disease and } \\
\text { gastrointestinal motility disorders }\end{array}$ \\
\hline
\end{tabular}

Figure 2 Multidisciplinary management of patients with congenital central hypoventilation syndrome.

Abbreviations: CCHS, congenital central hypoventilation syndrome; DP, diaphragm pacing; NPPV, noninvasive positive pressure ventilation; PHOX2B, paired-like homeobox 2B.

(PPV-T), noninvasive positive pressure ventilation (NPPV), negative pressure ventilation, and/or diaphragm pacing (DP). The goal of respiratory management is to provide AV to ensure adequate ventilation and oxygenation. This requires an individualized approach since the ventilatory requirement of each patient may vary during sleep and wakefulness, with growth, physical activity, and during illness. ${ }^{3,27}$ In contrast to children requiring home mechanical ventilation for other 
indications, patients with CCHS lack subjective and objective responses to hypercapnia and hypoxemia and do not manifest signs of increased work of breathing such as tachypnea and chest retractions. Therefore, they always require vigilant monitoring by skilled caregivers, pulse oximetry, and $\mathrm{ETCO}_{2}$ monitoring.

\section{Positive Pressure Ventilation via Tracheostomy}

At initial diagnosis of CCHS in infancy, the American Thoracic Society (ATS) clinical policy statement recommends PPV-T to optimize ventilation, oxygenation, and neurocognitive outcomes. Although studies have reported infants and children with CCHS managed exclusively with NPPV without ever receiving a tracheostomy, the ATS statement reports that PPV-T in the first few years of life is associated with better oxygenation and neurocognitive outcomes. ${ }^{1}$ Once the CCHS diagnosis is established in infancy, tracheostomy must be performed to deliver positive pressure ventilation. Pressure-controlled ventilation is preferred since it can compensate for variable tracheostomy leaks. ${ }^{1,3}$ Due to inadequate respiratory effort during sleep, patients may not trigger the ventilator with spontaneous breaths. Therefore, the ventilator settings should include an age-appropriate respiratory rate to ensure adequate breath delivery. Ventilator settings are adjusted to maintain $\mathrm{SpO}_{2} \geq 95 \%$ and $\mathrm{ETCO}_{2}$ between $35-40 \mathrm{~mm} \mathrm{Hg} .{ }^{1,3}$ In addition, visual and audible alarms should be programmed on the portable home ventilator for circuit disconnection and high and low volumes/ pressures. Despite internal ventilator alarms, patients should use pulse oximetry with alarms set for low oxygen saturation and abnormal heart rate for age.

At our institution, we provide full-time PPV-T for infants to optimize oxygenation, ventilation, and neurocognitive outcomes. Later in infancy or during early childhood, their respiratory pattern may evolve to sustain adequate ventilation during wakefulness likely due to maturation of the respiratory system. ${ }^{7,12}$ To assess the ventilatory requirement of the child and ability to breathe spontaneously during wakefulness, ventilator sprint weaning is performed by disconnecting $\mathrm{AV}$ for a brief duration (generally 30-60 minutes) under close medical supervision with pulse oximetry and $\mathrm{ETCO}_{2}$ monitoring. If there is hypoxemia or hypoventilation, sprint weaning is discontinued. If the patient tolerates a brief duration off $\mathrm{AV}$, then a sprint weaning progression plan is provided to gradually advance duration off $\mathrm{AV}$ with pulse oximetry and $\mathrm{ETCO}_{2}$ monitoring. The caregivers are instructed to have close follow up with the pulmonologist and to always use AV during sleep.

When feasible, relatively smaller and cuffless tracheostomy tubes are preferred as they permit an expiratory leak to facilitate speech using a speaking valve and may reduce the risk for tracheomalacia. Endoscopic evaluation of the airway and tracheostomy tube sizing must be periodically performed by a pediatric otolaryngologist. The ventilator settings should also be periodically evaluated with growth to ensure optimal oxygenation and ventilation. ${ }^{27}$ Programming a secondary ventilator setting with an increased respiratory rate and/or peak inspiratory pressure may be beneficial to provide better gas exchange during illness. Limitations of PPV-T include increased burden of care, speech delay, and tracheostomy-related complications such as infections, bleeding, obstruction, granulomas, and accidental decannulation. ${ }^{3}$

\section{Noninvasive Positive Pressure Ventilation}

The major benefit of NPPV is liberation from tracheostomy. NPPV can be considered in children with CCHS if they are cooperative, motivated, generally aged $\geq 6$ years, require AV only during sleep, and have a relatively stable medical course not requiring full-time AV with respiratory infections. ${ }^{3,28} \mathrm{NPPV}$ is also usually instituted in patients with lateonset CCHS presenting later in childhood or adulthood and requiring AV only during sleep. ${ }^{3,5}$ NPPV is delivered using a nasal mask, face mask, or nasal pillows using bilevel positive airway pressure (BPAP) or average-volume assured pressure support. The spontaneous timed mode or timed mode with an age appropriate respiratory rate should be used. ${ }^{28}$ Spontaneous mode should not be used as patients cannot increase their spontaneous respiratory rate in response to hypoxemia and hypercapnia. Continuous positive airway pressure (CPAP) therapy should not be used because it cannot support ventilation and deliver a respiratory rate. Portable home ventilators are recommended for NPPV therapy rather than BPAP devices that are not designed for life support. ${ }^{1,3}$ The inspiratory positive airway pressure (IPAP) and expiratory positive airway pressure (EPAP) are adjusted to maintain a sufficient pressure support (difference between IPAP and EPAP) to provide optimal tidal volumes. Ideal NPPV settings should be established during polysomnography to ensure adequate oxygenation and ventilation in different sleep stages per current titration guidelines. ${ }^{29}$ 
There are case studies reporting the use of NPPV from infancy without tracheostomy placement. However, there are no studies that have compared outcomes of children managed exclusively with NPPV from infancy versus PPV-T. During infancy and early childhood, patients may be relatively unstable and require full-time AV during respiratory infections that may be challenging to manage solely with NPPV. Other challenges with early institution of NPPV include finding a secure and well-fitting mask, prompt initiation of NPPV during daytime naps, and midface hypoplasia from mask use. Patients using NPPV require vigilant monitoring and supervision by skilled caregivers to prevent adverse consequences from mask dislocation. ${ }^{1,3}$

NPPV may permit tracheostomy decannulation (TD) in stable patients with CCHS requiring PPV-T only during sleep. ${ }^{1}$ Patients may seek TD to reduce burden of care, morbidities, tracheostomy-related complications, and for psychosocial factors. We reported that a systematic multidisciplinary algorithm may optimize successful transition from PPV-T to NPPV and TD. ${ }^{28}$ When eligible patients are identified, we utilize a multidisciplinary approach that includes collaboration between the patient and parents, pulmonologist, sleep physician, sleep psychologist, and otolaryngologist (Figure 3). The patient and parents are counseled on the risks and benefits of NPPV. NPPV mask fitting is performed, and motivated children are evaluated by the sleep psychologist, sleep physician, respiratory therapist, and otolaryngologist. Endoscopic airway evaluation is performed in the operating room, and the tracheostomy tube may be downsized to facilitate daytime tracheostomy capping trials. During the multidisciplinary clinic visit, low pressure NPPV trial using the patient's own mask is initiated for acclimation with the tracheostomy tube capped. When the patient tolerates tracheostomy tube capping all day during wakefulness and daytime NPPV trials at home, polysomnography with NPPV titration and downsized capped tracheostomy tube using the patient's own mask is performed to identify optimal NPPV settings. An experienced pediatric sleep lab respiratory therapist is critical to ensure a successful polysomnography with NPPV titration. ${ }^{29}$ TD is performed during a hospitalization when optimal NPPV settings are identified during polysomnography. Patients require vigilant monitoring by trained caregivers and home healthcare nursing in the initial period following TD to ensure there is no mask dislocation or excessive air leak from the tracheostomy stoma during sleep on NPPV.

NPPV-related complications include midface hypoplasia, dental malocclusion, and skin ulceration. Patients using NPPV should be periodically evaluated by a craniofacial physician to monitor facial growth. In addition, the mask fit should be routinely assessed with growth to avoid a poor-fitting interface which can interfere with treatment. Annual polysomnography with NPPV titration should be performed to ensure optimal settings are identified with growth. ${ }^{1,3}$

\section{Diaphragm Pacing}

DP is a modality of AV in CCHS that generates respiration using the patient's own diaphragm by electrical stimulation of the phrenic nerves. The components of the DP system include 1) an external battery-operated transmitter, 2) external antennae that are secured over the subcutaneous receivers, 3) subcutaneously implanted radiofrequency receivers, and 4)

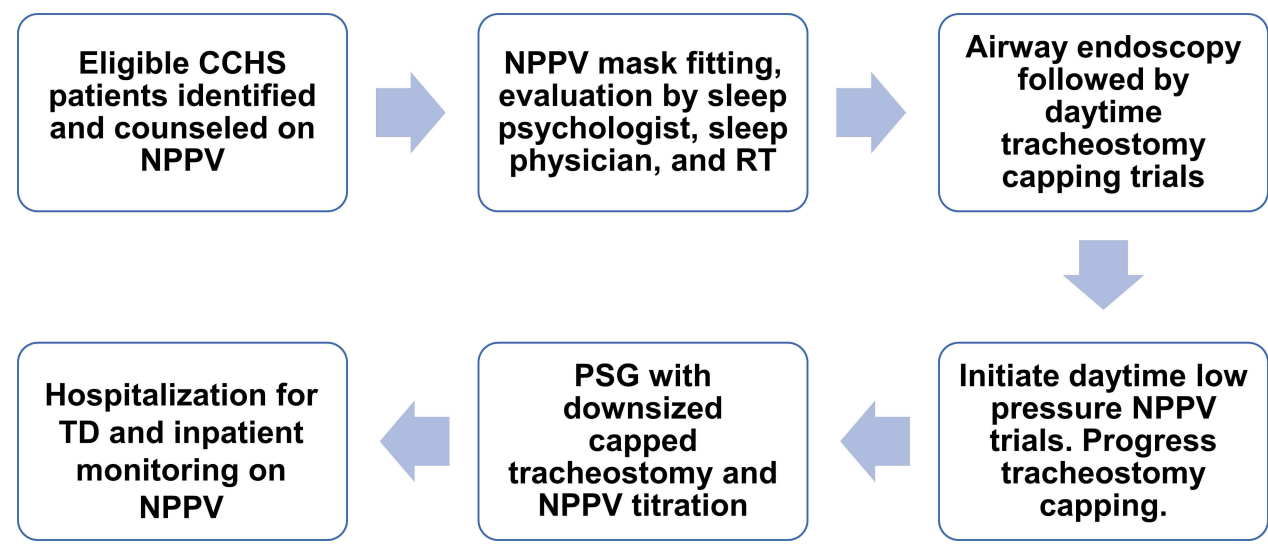

Figure 3 Algorithm for tracheostomy decannulation to noninvasive positive pressure ventilation in congenital central hypoventilation syndrome. ${ }^{28}$ Abbreviations: CCHS, congenital central hypoventilation syndrome; NPPV, noninvasive positive pressure ventilation; PSG, polysomnography; RT, respiratory therapist; TD, tracheostomy decannulation. 
electrodes that are surgically implanted on the phrenic nerves (Figure 4). The transmitter generates a train of pulses that are transmitted to the antennae taped on the skin above the subcutaneous receivers. The subcutaneous receivers convert the radiofrequency signal from the transmitter into an electrical current that is conducted to the phrenic nerve electrodes. Electrical stimulation of the phrenic nerves leads to diaphragm contraction that generates a breath. ${ }^{1,6}$ The strength of the diaphragm contraction corresponds to the generated tidal volume that can be controlled using the transmitter amplitude settings. The frequency of impulse generation can also be controlled using the transmitter and corresponds to the patient's respiratory frequency. ${ }^{30}$

In patients requiring full-time AV, DP allows freedom from PPV-T during the day. Since the DP system is small, compact, portable, and tubing-free, it permits more mobility allowing patients to participate in school and other activities without being connected to a ventilator circuit. In stable CCHS patients requiring PPV-T only during sleep, DP may permit TD. ${ }^{31}$ At our institution, we consider DP in patients requiring full-time AV so that DP can be used during the day and PPV-T during sleep. DP with potential TD is considered for eligible patients aged $\geq 4$ years, requiring AV only during sleep, with minimal or no lung disease, no obesity, and with a stable medical course not requiring full-time AV with respiratory infections. An ideal candidate should have intact phrenic nerves and normal diaphragm function that is assessed by a diaphragm ultrasound or fluoroscopy off AV to demonstrate spontaneous diaphragm movement. ${ }^{3}$ When eligible patients seeking DP are identified, a coordinated multidisciplinary approach is utilized collaborating with the pulmonologist, otolaryngologist, cardiologist, surgeon, anesthesiologist, intensivist, and sleep physician. Following an initial discussion of the risks and benefits of DP with the pulmonologist, the patient is evaluated by the pediatric surgeon, anesthesiologist, and cardiologist for preoperative planning, and insurance approval is obtained for the DP system and surgery (Figure 5).

Bilateral electrodes are surgically implanted on the phrenic nerves thoracoscopically. ${ }^{32}$ The surgical steps include identification of the phrenic nerves, implantation of the electrodes on the phrenic nerves, testing the DP intraoperatively to ensure electrode stimulation leads to diaphragm contraction, and subcutaneous implantation of the receivers in the lower chest or abdomen. ${ }^{33}$ In addition to general anesthesia administration, anesthesia management during surgery includes maintaining the airway with a double-lumen endotracheal tube or sequential mainstem intubation for lung

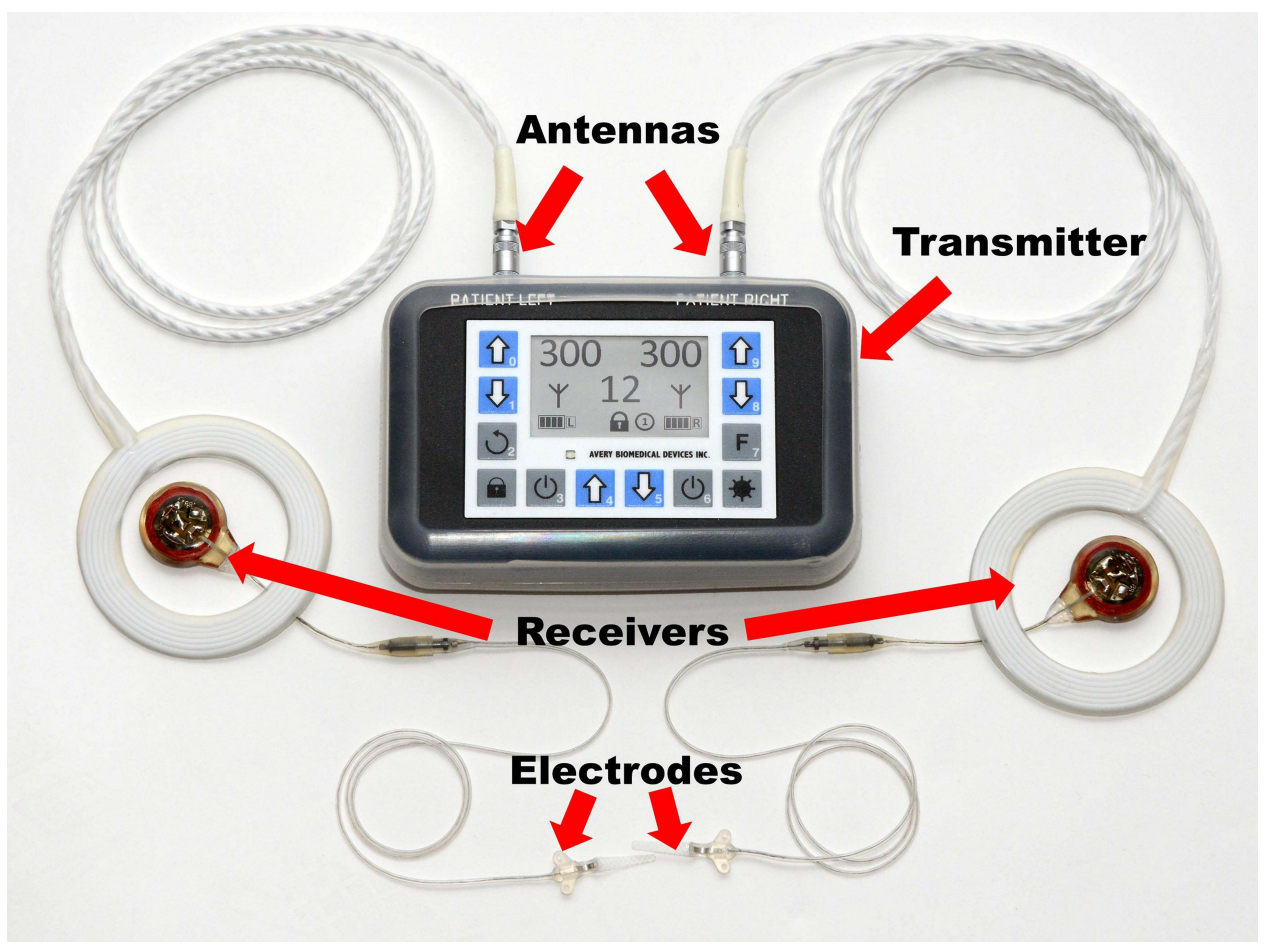

Figure 4 Diaphragm pacemaker system. Avery diaphragm pacemaker system and Spirit transmitter. Printed with permission by Avery biomedical devices (Commack, NY, USA). 


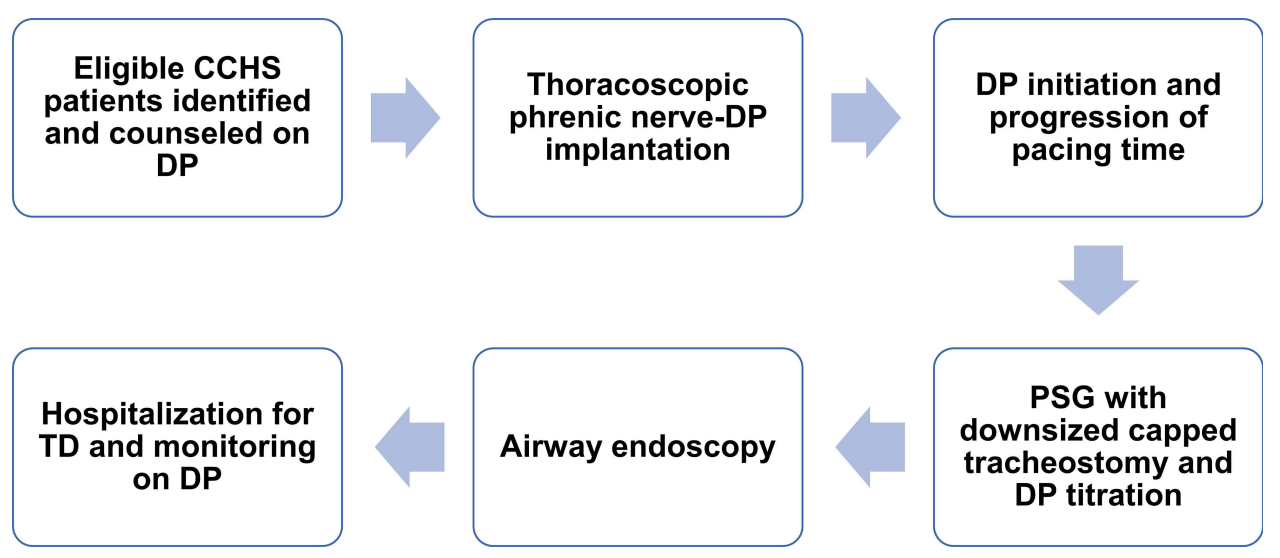

Figure 5 Algorithm for tracheostomy decannulation to diaphragm pacing in congenital central hypoventilation syndrome. ${ }^{31}$

Abbreviations: CCHS, congenital central hypoventilation syndrome; DP, diaphragm pacing; PSG, polysomnography; TD, tracheostomy decannulation.

isolation to provide thoracoscopic access and ensuring adequate $\mathrm{AV}$ is provided. ${ }^{34}$ The DP is not used immediately after surgery and the patient receives mechanical AV during postoperative recovery in the intensive care unit.

DP is initiated around 4 weeks following surgery to allow for wound healing and stabilization of tissue reaction around the phrenic nerve electrodes. DP is initiated with the child awake and off PPV-T with continuous pulse oximetry and $\mathrm{ETCO}_{2}$ monitoring. The amplitude settings are gradually increased on both sides until a good chest rise and diaphragm contraction are observed. $\mathrm{SpO}_{2}$ and $\mathrm{ETCO}_{2}$ are monitored with increase in the amplitude settings. When DP is initiated, it is limited to around 60-90 minutes per day since it can lead to diaphragm fatigue with the same amplitude settings resulting in reduced diaphragm contraction. Once DP is initiated and settings are identified, a DP progression plan is provided for the patient to advance duration on DP at home with pulse oximetry and $\mathrm{ETCO}_{2}$ monitoring. DP is gradually increased by 30 minutes per day each week until the patient tolerates full pacing that entails DP during wakefulness in patients requiring full-time AV or DP during sleep in patients requiring AV only during sleep. This gradual progression in DP allows for aerobic training of the diaphragm muscle fibers to sustain the entire duration of DP. During the period of progression in DP, the patient's ventilator is used for AV when off DP. ${ }^{35}$

When patients requiring AV only during sleep can use DP throughout the duration of sleep, polysomnography with uncapped tracheostomy tube and DP titration is performed to identify optimal settings for adequate oxygenation and ventilation. ${ }^{30}$ In patients requiring AV only during sleep, TD can be considered after successful overnight DP with uncapped tracheostomy tube for $\geq 3$ months (Figure 5). ${ }^{31}$ Subsequently, polysomnography with DP titration and downsized capped tracheostomy tube is performed by an experienced sleep lab respiratory therapist. If optimal DP settings are identified to maintain adequate oxygenation and ventilation without obstructive sleep apnea (OSA), then an endoscopic airway evaluation is performed by the otolaryngologist followed by hospitalization for TD. During the polysomnography, if OSA is identified, the DP amplitude settings may need to be reduced to alleviate OSA. ${ }^{36}$ However, if OSA persists with derangements in oxygenation and ventilation despite reducing the DP amplitude settings, the patient may require an adenotonsillectomy and drug-induced sleep endoscopy to identify upper airway obstruction with DP. ${ }^{31,36-38}$ Polysomnography with DP titration and downsized capped tracheostomy tube should be repeated before proceeding with TD. ${ }^{31}$ OSA can occur in some patients using DP due to the absence of synchronous contraction of the diaphragm and upper airway skeletal muscles. Although OSA can be alleviated in some patients by adjustments in DP settings during polysomnography, severe OSA can occur in some patients that may be preclude TD. ${ }^{36,38}$ In addition, obesity may hinder successful DP due to impaired signal transmission between the antennae and subcutaneous receivers by increased adipose tissue. ${ }^{36}$

In children, DP is limited to 12-14 hours per day to prevent diaphragm fatigue and consequent inadequate ventilation. ${ }^{3}$ Other limitations include potential damage to internally implanted components requiring surgical replacement, positional variation in gas exchange, and inability to undergo MRI scans. ${ }^{1,33,39}$ During respiratory infections requiring increased duration of $\mathrm{AV}$, DP may not provide sufficient ventilatory support, and alternative forms of AV may 
need to be utilized. ${ }^{6}$ Since CCHS is a rare disease and DP is not a widely adopted modality of AV, only a few hospitals and medical professionals are experienced with managing patients using DP and performing polysomnography with DP titration, limiting their management to a few CCHS centers with expertise.

\section{Negative Pressure Ventilation}

Negative pressure ventilators generate a negative pressure outside the chest and abdomen that expands the chest, thereby promoting inspiration. Negative pressure ventilators include the chest shell (cuirass), wrap, and tank ventilators. ${ }^{1}$ The negative pressure and ventilator rate can be programmed in the ventilator. The negative pressure is related to the tidal volume; however, this may be limited by air leaks around the wrap or cuirass. Although effective ventilation can be provided in some children without a tracheostomy, airway occlusion can occur during sleep, limiting its use. Additional limitations include reduced portability, difficulty sleeping in the supine position, and skin irritation. ${ }^{1,3}$ Owing to these challenges, negative pressure ventilation is rarely used in patients with CCHS. ${ }^{3,6}$

\section{Sleep Medicine Management}

A sleep medicine team comprising of a sleep physician, sleep psychologist, and respiratory therapist can be helpful in the successful transition of patients from PPV-T to NPPV and TD (Figure 3) ${ }^{28}$ In patients pursuing NPPV for TD, the sleep physician reviews the use of NPPV with the patient and parents, assesses the mask, and monitors tolerance of low pressure NPPV daytime trials for desensitization and readiness for capped tracheostomy polysomnography with NPPV titration. Pediatric sleep psychologists are involved in consultative and intervention roles by assisting patients transition from PPV-T to NPPV. The psychologist partners with the patient, family, and medical team to explore and assist with barriers that may hinder successful transition to NPPV such as sleep problems, anxiety related to NPPV, motivation, and social factors. In addition, the psychologist also develops individualized plans to assist patients with this transition including acclimation and adherence to NPPV, improving sleep hygiene, and to optimize mask fit along with the respiratory therapist. Following TD, the psychologist continues to work with the patient and family to maintain optimal NPPV adherence. During each clinic visit, NPPV adherence is reviewed using BPAP compliance reports and the patient is assessed for issues that may interfere with ongoing adherence such as mask and equipment-related, social, individual, and medical challenges. ${ }^{28}$

Polysomnography is an important diagnostic and management tool in CCHS. Apart from the diagnostic utility of polysomnography in the evaluation of suspected cases of CCHS, it is also utilized to: 1) assess and titrate ventilator settings to maintain adequate oxygenation and ventilation in patients using PPV-T, NPPV, and DP; and 2) assess readiness for TD during a capped tracheostomy study with NPPV and DP titration. ${ }^{21,27,28,30,39}$ An experienced pediatric sleep-trained respiratory therapist familiar with the unique aspects of sleep abnormalities in CCHS is vital in ensuring a successful polysomnography with different modalities of $\mathrm{AV}^{27,28}$

\section{Otolaryngologic Management}

Pediatric otolaryngologists play a key role in the multidisciplinary care of CCHS children with a tracheostomy. This includes tracheostomy placement, surveillance airway endoscopy, evaluating the tracheostomy tube size, and collaborative care of patients pursuing TD. At our institution, we encourage the use of speaking valves following tracheostomy tube manometry to assess safety and tolerance. The tracheostomy tube size needs to be periodically assessed and upsized with growth to maintain adequate ventilation. Surveillance bronchoscopy under anesthesia is performed periodically to assess the airway anatomy, tracheostomy tube size and position, and for airway granulomas. ${ }^{1,3}$ Procedures requiring anesthesia are generally coordinated with other annual surveillance evaluations such as postoperative hospitalization for assessment of ventilator settings, imaging studies for NCT, and cardiovascular studies. In the outpatient setting, the otolaryngologist manages tracheostomy-dependent patients in our multidisciplinary technology-dependent clinic consisting of a pulmonologist, respiratory therapist, nurse coordinator, and social worker. ${ }^{28}$

In patients pursuing $\mathrm{TD}$, the otolaryngologist collaborates with the sleep physician, sleep psychologist, and pulmonologist to determine timing of bronchoscopy, downsizing the tracheostomy tube, and initiating daytime tracheostomy tube capping trials. ${ }^{28,40}$ This multidisciplinary approach may optimize successful transition to NPPV or DP and facilitate 
$\mathrm{TD}^{28}$ In patients using DP during sleep with persistent OSA on the capped tracheostomy polysomnography despite adjustments in DP settings, drug-induced sleep endoscopy with DP may be beneficial to identify areas of upper airway obstruction followed by targeted surgical interventions. ${ }^{37,38}$ During this procedure, the upper airway anatomy and dynamics are examined under sedation with a range of DP settings. Following TD, persistent tracheocutaneous fistula may be a common sequela that requires surgical closure. ${ }^{28}$

\section{Cardiovascular Management}

In patients with CCHS, life-threatening cardiac arrhythmias and pulmonary hypertension can manifest at different ages. The current recommendations include annual echocardiography to assess for pulmonary hypertension and ambulatory cardiac monitoring with a minimum monitoring time of 72 hours to detect cardiac arrhythmias and sinus pauses. ${ }^{1,3}$ There is an association between the size of polyalanine expansion in patients with PARMs and the duration of R-R interval on ambulatory cardiac monitoring requiring cardiac pacemaker implantation. ${ }^{24} \mathrm{~A}$ recent study concluded that regardless of the PHOX2B genotype, CCHS patients are at risk for significant sinus pauses and may present with syncope, palpitations, chest pain, and dizziness. ${ }^{22}$ Symptomatic patients with recurrent syncope and R-R interval $\geq 3$ seconds should undergo cardiac pacemaker implantation. Asymptomatic patients with R-R interval $\geq 3$ seconds should consider cardiac pacemaker implantation based on other factors and shared decision making. Moreover, some symptomatic patients without significant sinus pauses may still require implantation of a cardiac pacemaker. ${ }^{3,22}$ Annual echocardiogram and ambulatory cardiac monitoring or cardiac pacemaker interrogation are generally performed during an inpatient hospitalization to coordinate care.

Some patients may require both a cardiac pacemaker and a diaphragm pacer that can be used together. To avoid potential electromagnetic interference with the DP, bipolar cardiac pacemakers are recommended. ${ }^{1}$ Placement of cardiac pacemaker leads and programming should be done to prevent inhibition of cardiac pacing from far-field signals from DP leads. In patients undergoing procedures under anesthesia, cardiac arrhythmias can occur intraoperatively. ${ }^{4}$ Therefore, preoperative electrocardiogram, echocardiogram, and evaluation by a cardiologist for patients with implanted cardiac pacemakers are indicated.

\section{Oncological Management}

CCHS is a cancer predisposition syndrome with associated risk for the development of NCTs including neuroblastoma and ganglioneuroblastoma. The cancer risk is extremely significant in those patients with NPARMs, estimated at 45-50\%. Patients with longer PARMs (20/28 - 20/33) also carry an increased likelihood to develop cancer, but only in the range of $1-2 \% .{ }^{1,41}$ Further, neuroblastomas associated with genetic syndromes like CCHS generally occur earlier than sporadic cases with a mean age of diagnosis of 9 months versus 2-3 years, respectively. ${ }^{41,42}$ Tumor surveillance guidelines for the above patients with CCHS have been proposed with the goal of early identification and intervention (Table 1). These studies include biochemical and imaging studies in the first 10 years of life, carried out more frequently from ages 0 to 6 years (every 3 months) and less frequently from age 6 to 10 years (every 6 months). Generally, no tumor surveillance is recommended after the age of 10 years, unless otherwise recommended by the oncologist. Adrenal or abdominal tumors are screened for using abdominal ultrasound. Mediastinal and other sympathetic chain tumors are screened for using chest radiography. Urine catecholamines (homovanillic acid and vanillylmandelic acid) are added at each surveillance timepoint to help identify tumors potentially not well-visualized on the imaging studies. ${ }^{1,3}$ At our institution, surveillance imaging and urine studies may be performed during a hospitalization for assessment of ventilator settings or combined with a postoperative hospitalization. They are also done easily as an outpatient visit and without need for sedation, realizing the complexity sedation poses for patients with CCHS. When a tumor is identified, surgical excision or biopsy is required to ascertain the diagnosis and guide the development of a treatment plan. The utility of serial imaging in patients with $P H O X 2 B$ genotypes PARM 20/24 - 20/27 is unclear. ${ }^{1}$

\section{Anesthesia Management}

In patients with CCHS, general anesthetic medications, sedatives, and opioids can cause respiratory depression and worsen hypoventilation. Intraoperative risks include cardiac arrhythmias, hypoxemia, hypercapnia, and hypothermia. 
Therefore, perioperative planning should include a multidisciplinary discussion between the surgeon, pulmonologist, cardiologist, and anesthesiologist. ${ }^{4,34}$ A thorough preoperative assessment is vital in formulating a safe anesthetic plan for different procedures. Cardiac arrhythmias, especially bradyarrhythmia, can occur intraoperatively and some patients may have implanted cardiac pacemakers. ${ }^{4}$ Obtaining a preoperative electrocardiogram, echocardiogram, and cardiology consultation to assist with the perioperative management of cardiac pacemakers is important. The patient's baseline home ventilatory support, postoperative ventilatory support plan, and hospitalization for postoperative monitoring should be discussed with the pulmonologist.

Routinely administered preoperative sedatives and anxiolytics such as opioids and benzodiazepines will lead to respiratory depression and hypoventilation. Therefore, it is preferable to avoid preoperative sedatives. However, if preoperative sedatives are required, the patient should receive AV with continuous pulse oximetry until the patient is taken into the operating room for the surgery. Intraoperative management includes providing adequate AV to prevent hypoxemia and hypercapnia, electrocardiographic monitoring and avoidance of drugs that may worsen bradycardia, and hemodynamic monitoring. Both intravenous and volatile medications have been used safely, although recommendations steer towards using short-acting agents, when feasible. ${ }^{4}$ Anticholinergics such as glycopyrrolate and atropine should be available for potential bradycardia. If neuromuscular blockade is required to facilitate surgery, full reversal should be done at the conclusion of the procedure. ${ }^{4}$ Postoperatively, patients will require AV due to respiratory depression from anesthesia. Postoperative management includes providing AV with continuous pulse oximetry, capnography, and potential hospitalization for ongoing monitoring.

Special considerations in patients undergoing thoracoscopic bilateral phrenic nerve implantation include lung isolation either by a double lumen endotracheal tube (generally aged $>8$ years), sequential mainstem intubation (generally aged $<8$ years), or bronchial blocker, increased AV settings for single-lung ventilation, and reversal of neuromuscular blockade, if used, for intraoperative testing of the phrenic nerve electrodes. ${ }^{34}$ Postoperative considerations include providing continuous $\mathrm{AV}$ and airway clearance therapies for lung recruitment in the intensive care unit. Opioid analgesics should not be excluded from the postoperative pain management plan since patients receive continuous AV. Adjuncts such as non-steroidal anti-inflammatory drugs should be considered if there is no concern with surgical bleeding. ${ }^{34}$ Lastly, anesthesiologists should maintain a high index of suspicion for undiagnosed late-onset CCHS in patients with unexplained postoperative respiratory failure. ${ }^{5}$

\section{Genetics Management}

Identifying a pathogenic variant in $P H O X 2 B$ not only confirms the diagnosis of CCHS, but also aids in predicting the clinical severity and disease manifestations, provides guidance for surveillance evaluations, and facilitates molecular testing of at-risk family members. ${ }^{1,9}$ Currently, most molecular diagnostic labs use short-read next generation sequencing (NGS)-based assays for genetic testing, including gene panel testing or exome sequencing. However, this technology alone cannot detect polyalanine repeat expansions of PARMs that cause the majority of CCHS, and it is not sensitive for deletion, duplication, and mosaicism detection. Thus, when ordering molecular testing of $P H O X 2 B$, it is important to know this limitation and communicate with the lab regarding test strategies to identify PARMs, NPARMs, and copy number variants. CCHS is an autosomal dominant inherited condition. Although in most cases, variants in $P H O X 2 B$ occur de novo, some were reported to be inherited from one of the parents. So targeted molecular testing for the familial variants in $P H O X 2 B$ is recommended for both parents of the affected child. ${ }^{1}$ Notably, up to $10-25 \%$ of affected individuals have an asymptomatic parent mosaic for the familial $P H O X 2 B$ variant. ${ }^{43}$ Relatively asymptomatic parents with $P H O X 2 B$ variants can manifest symptoms later in life and require periodic evaluations for respiratory and cardiac disturbances. ${ }^{19,20}$

Genetic counseling is essential for patients with CCHS and their parents since there is a $50 \%$ recurrence risk with each child. Parental testing is important; however, even if it is negative, low level somatic or germline mosaicism in $P H O X 2 B$ cannot be ruled out. For family planning, because of the relatively high frequency of parental mosaicism in $P H O X 2 B$, the fetus should be considered at risk for CCHS even with negative parental PHOX2B tests. Therefore, prenatal genetic counseling is highly recommended, and prenatal testing should be considered. Prenatal testing and 
knowledge of the anticipated infant's CCHS status can aid in family planning and preparation of the medical team for delivery room and neonatal care. ${ }^{1,9}$

\section{Other Multidisciplinary Management}

The gastrointestinal manifestations in CCHS include HD, esophageal dysmotility, gastroesophageal reflux disease (GERD), dysphagia requiring gastrostomy tube feeding, and constipation. HD occurs in around $20 \%$ of patients and is more prevalent in patients with NPARMs than those with PARMs. ${ }^{1,3}$ Most patients are diagnosed with HD during early infancy when they present with failure to pass meconium on the first day of life, abdominal distension, emesis, and constipation. In patients with suspected HD, barium enema or manometry and rectal biopsy should be performed to diagnose HD. ${ }^{1}$ Despite surgical resection of the affected gut, gastrointestinal dysmotility may persist requiring management by a gastroenterologist. ${ }^{23}$ Patients with symptoms of swallowing dysfunction may be at risk for aspiration and a videofluoroscopic swallow study may be indicated. Patients should be screened for symptoms of GERD, dysphagia, and constipation and pertinent evaluations should be performed in conjunction with an experienced gastroenterologist.

Ocular disorders such as strabismus, pupillary abnormalities, convergence insufficiency, and ptosis are prevalent in CCHS. Comprehensive ocular evaluation by an ophthalmologist should be performed when CCHS is diagnosed and annually thereafter. Early recognition and treatment of ocular disorders can minimize learning problems. ${ }^{1,3}$ Children with CCHS are at risk for neurodevelopmental delays due to $P H O X 2 B$ gene dysfunction and/or recurrent hypoxemia and hypoventilation. In addition to optimizing the patient's ventilator settings to maintain adequate gas exchange, annual comprehensive neurocognitive testing should be performed and therapies should be instituted to maximize the child's neurocognitive potential. ${ }^{1,25}$ With advances in CCHS diagnosis and management, patients using different modalities of AV are surviving into adulthood and transitioning to adult pulmonology and sleep physicians where comprehensive multidisciplinary care may not be available. Therefore, adult clinicians should become familiar with the management of CCHS, incorporate capnography during polysomnography, and collaborate with an experienced CCHS center. ${ }^{3,6}$

\section{Conclusion}

CCHS is a rare and lifelong disorder affecting infants, children, and adults. In addition to respiratory dysfunction, several organs are affected including the cardiovascular, gastrointestinal, and neurologic systems. Each area of care influences the overall health of the patient and impacts long-term outcomes. ${ }^{1}$ Therefore, a comprehensive multidisciplinary approach may ensure the best possible outcomes. Furthermore, with increased survival into adulthood, there is a necessity in ensuring availability of high-quality multidisciplinary care for adults with CCHS. ${ }^{3}$ Advances in the management of CCHS, knowledge of $P H O X 2 B$ gene pathways, and ongoing clinical and genetic studies may shed light on the journey to identifying a potential cure for CCHS.

\section{Disclosure}

The authors report no conflicts of interest in this work.

\section{References}

1. Weese-Mayer DE, Berry-Kravis EM, Ceccherini I, Keens TG, Loghmanee DA, Trang H. An official ATS clinical policy statement: congenital central hypoventilation syndrome: genetic basis, diagnosis, and management. Am J Respir Crit Care Med. 2010;181(6):626-644. doi:10.1164/ recm.200807-1069ST

2. Mellins RB, Balfour HHJ, Turino GM, Winters RW. Failure of automatic control of ventilation (Ondine's curse). Report of an infant born with this syndrome and review of the literature. Medicine. 1970;49(6):487-504. doi:10.1097/00005792-197011000-00003

3. Trang H, Samuels M, Ceccherini I, et al. Guidelines for diagnosis and management of congenital central hypoventilation syndrome. Orphanet $J$ Rare Dis. 2020;15(1):252. doi:10.1186/s13023-020-01460-2

4. Basu SM, Chung FF, AbdelHakim SF, Wong J. Anesthetic considerations for patients with congenital central hypoventilation syndrome: a systematic review of the literature. Anesth Analg. 2017;124(1):169-178. doi:10.1213/ANE.0000000000001470

5. Hino A, Terada J, Kasai H, et al. Adult cases of late-onset congenital central hypoventilation syndrome and paired-like homeobox 2B-mutation carriers: an additional case report and pooled analysis. J Clin Sleep Med. 2020;16(11):1891-1900. doi:10.5664/jcsm.8732

6. Kasi AS, Perez IA, Kun SS, Keens TG. Congenital central hypoventilation syndrome: diagnostic and management challenges. Pediatric Health Med Ther. 2016;7:99-107. doi:10.2147/PHMT.S95054 
7. Kasi AS, Li H, Jurgensen TJ, Guglani L, Keens TG, Perez IA. Variable phenotypes in congenital central hypoventilation syndrome with PHOX2B nonpolyalanine repeat mutations. J Clin Sleep Med. 2021;17(10):2049-2055. doi:10.1007/s11325-021-02368-2

8. Marcus CL, Jansen MT, Poulsen MK, et al. Medical and psychosocial outcome of children with congenital central hypoventilation syndrome. $J$ Pediatr. 1991;119(6):888-895. doi:10.1016/S0022-3476(05)83038-0

9. Bishara J, Keens TG, Perez IA. The genetics of congenital central hypoventilation syndrome: clinical implications. Appl Clin Genet. 2018;11:135144. doi:10.2147/TACG.S140629

10. Huang J, Colrain IM, Panitch HB, et al. Effect of sleep stage on breathing in children with central hypoventilation. J Appl Physiol. 2008;105(1):4453. doi:10.1152/japplphysiol.01269.2007

11. Marcus CL, Bautista DB, Amihyia A, Ward SL, Keens TG. Hypercapneic arousal responses in children with congenital central hypoventilation syndrome. Pediatrics. 1991;88(5):993-998.

12. Perez IA, Keens TG. Peripheral chemoreceptors in congenital central hypoventilation syndrome. Respir Physiol Neurobiol. 2013;185(1):186-193. doi:10.1016/j.resp.2012.10.008

13. Paton JY, Swaminathan S, Sargent CW, Keens TG. Hypoxic and hypercapnic ventilatory responses in awake children with congenital central hypoventilation syndrome. Am Rev Respir Dis. 1989;140(2):368-372. doi:10.1164/ajrccm/140.2.368

14. Silvestri JM, Weese-Mayer DE, Flanagan EA. Congenital central hypoventilation syndrome: cardiorespiratory responses to moderate exercise, simulating daily activity. Pediatr Pulmonol. 1995;20(2):89-93. doi:10.1002/ppul.1950200207

15. Paton JY, Swaminathan S, Sargent CW, Hawksworth A, Keens TG. Ventilatory response to exercise in children with congenital central hypoventilation syndrome. Am Rev Respir Dis. 1993;147(5):1185-1191. doi:10.1164/ajrccm/147.5.1185

16. Gozal D, Marcus CL, Ward SL, Keens TG. Ventilatory responses to passive leg motion in children with congenital central hypoventilation syndrome. Am J Respir Crit Care Med. 1996;153(2):761-768. doi:10.1164/ajrccm.153.2.8564130

17. Gozal D, Simakajornboon N. Passive motion of the extremities modifies alveolar ventilation during sleep in patients with congenital central hypoventilation syndrome. Am J Respir Crit Care Med. 2000;162(5):1747-1751. doi:10.1164/ajrccm.162.5.2005012

18. Shea SA, Andres LP, Paydarfar D, Banzett RB, Shannon DC. Effect of mental activity on breathing in congenital central hypoventilation syndrome. Respir Physiol. 1993;94(3):251-263. doi:10.1016/0034-5687(93)90022-3

19. Kasi AS, Kun SS, Keens TG, Perez IA. Adult with PHOX2B mutation and late-onset congenital central hypoventilation syndrome. J Clin Sleep Med. 2018;14(12):2079-2081. doi:10.5664/jcsm.7542

20. Kasi AS, Jurgensen TJ, Yen S, Kun SS, Keens TG, Perez IA. Three-generation family with congenital central hypoventilation syndrome and novel PHOX2B gene non-polyalanine repeat mutation. J Clin Sleep Med. 2017;13(7):925-927. doi:10.5664/jcsm.6670

21. Anand N, Leu RM, Simon D, Kasi AS. Recurrent apnoea and respiratory failure in an infant: congenital central hypoventilation syndrome with a novel PHOX2B gene variant. BMJ Case Rep. 2021;14(3):e239633. doi:10.1136/bcr-2020-239633

22. Laifman E, Keens TG, Bar-Cohen Y, Perez IA. Life-threatening cardiac arrhythmias in congenital central hypoventilation syndrome. Eur J Pediatr. 2020;179(5):821-825. doi:10.1007/s00431-019-03568-5

23. Balakrishnan K, Perez IA, Keens TG, Sicolo A, Punati J, Danialifar T. Hirschsprung disease and other gastrointestinal motility disorders in patients with CCHS. Eur J Pediatr. 2021;180(2):469-473. doi:10.1007/s00431-020-03848-5

24. Gronli JO, Santucci BA, Leurgans SE, Berry-Kravis EM, Weese-Mayer DE. Congenital central hypoventilation syndrome: PHOX2B genotype determines risk for sudden death. Pediatr Pulmonol. 2008;43(1):77-86. doi:10.1002/ppul.20744

25. Charnay AJ, Antisdel-Lomaglio JE, Zelko FA, et al. Congenital central hypoventilation syndrome: neurocognition already reduced in preschoolaged children. Chest. 2016;149(3):809-815. doi:10.1378/chest.15-0402

26. Children's healthcare of Atlanta diaphragm pacing. Available from: https://www.choa.org/medical-services/pulmonology/diaphragm-pacing. Accessed December 31, 2021.

27. Shah AS, Leu RM, Keens TG, Kasi AS. Annual respiratory evaluations in congenital central hypoventilation syndrome and changes in ventilatory management. Pediatr Allergy Immunol Pulmonol. 2021;34(3):97-101. doi:10.1089/ped.2021.0072

28. Kasi AS, Anand N, Harford K-L, et al. Tracheostomy decannulation to noninvasive positive pressure ventilation in congenital central hypoventilation syndrome. Sleep Breath. 2021. doi:10.1007/s11325-021-02368-2

29. Berry RB, Chediak A, Brown LK, et al. Best clinical practices for the sleep center adjustment of noninvasive positive pressure ventilation (NPPV) in stable chronic alveolar hypoventilation syndromes. J Clin Sleep Med. 2010;6(5):491-509.

30. Pino-Diaz L, Leu RM, Kasi AS. Images: polysomnographic artifacts in a child with congenital central hypoventilation syndrome. J Clin Sleep Med. 2020;16(12):2123-2125. doi:10.5664/jcsm.8816

31. Diep B, Wang A, Kun S, et al. Diaphragm pacing without tracheostomy in congenital central hypoventilation syndrome patients. Respiration. 2015;89(6):534-538. doi:10.1159/000381401

32. Shaul DB, Danielson PD, McComb JG, Keens TG. Thoracoscopic placement of phrenic nerve electrodes for diaphragmatic pacing in children. $J$ Pediatr Surg. 2002;37(7):974-978. doi:10.1053/jpsu.2002.33821

33. Nicholson KJ, Nosanov LB, Bowen KA, et al. Thoracoscopic placement of phrenic nerve pacers for diaphragm pacing in congenital central hypoventilation syndrome. J Pediatr Surg. 2015;50(1):78-81. doi:10.1016/j.jpedsurg.2014.10.002

34. Ballard HA, Leavitt OS, Chin AC, et al. Perioperative anesthetic management of children with congenital central hypoventilation syndrome and rapid-onset obesity with hypothalamic dysfunction, hypoventilation, and autonomic dysregulation undergoing thoracoscopic phrenic nervediaphragm pacemaker. Paediatr Anaesth. 2018;28(11):963-973. doi:10.1111/pan.13475

35. Chen ML, Tablizo MA, Kun S, Keens TG. Diaphragm pacers as a treatment for congenital central hypoventilation syndrome. Expert Rev Med Devices. 2005;2(5):577-585. doi:10.1586/17434440.2.5.577

36. Wang A, Kun S, Diep B, Davidson ward SL, Keens TG, Perez IA. Obstructive sleep apnea in patients with congenital central hypoventilation syndrome ventilated by diaphragm pacing without tracheostomy. J Clin Sleep Med. 2018;14(2):261-264. doi:10.5664/jcsm.6948

37. Baldassari CM, Lam DJ, Ishman SL, et al. Expert consensus statement: pediatric drug-induced sleep endoscopy. Otolaryngol Head Neck Surg. 2021;165(4):578-591. doi:10.1177/0194599820985000

38. Valika T, Chin AC, Thompson DM, et al. Airway obstruction during sleep due to diaphragm pacing precludes decannulation in young children with CCHS. Respiration. 2019;98(3):263-267. doi:10.1159/000501172 
39. Chada A, Leu RM, Perez IA, Esther CRJ, Kasi AS. Positional impairment of gas exchange during diaphragm pacing alleviated by increasing amplitude settings in congenital central hypoventilation syndrome. J Clin Sleep Med. 2020;16(3):459-462. doi:10.5664/jcsm.8232

40. Mitchell RB, Hussey HM, Setzen G, et al. Clinical consensus statement: tracheostomy care. Otolaryngol Head Neck Surg. 2013;148(1):6-20. doi: $10.1177 / 0194599812460376$

41. Kamihara J, Bourdeaut F, Foulkes WD, et al. Retinoblastoma and neuroblastoma predisposition and surveillance. Clin Cancer Res. 2017;23(13): e98-e106. doi:10.1158/1078-0432.CCR-17-0652

42. Mitchell SG, Pencheva B, Westfall E, Porter CC. Cancer predisposition in neonates and infants: recognition, tumor types, and surveillance. Clin Perinatol. 2021;48(1):1-14. doi:10.1016/j.clp.2020.11.001

43. Bachetti T, Parodi S, Di Duca M, Santamaria G, Ravazzolo R, Ceccherini I. Low amounts of PHOX2B expanded alleles in asymptomatic parents suggest unsuspected recurrence risk in congenital central hypoventilation syndrome. J Mol Med (Berl). 2011;89(5):505-513. doi:10.1007/s00109010-0718-y

\section{Publish your work in this journal}

The Journal of Multidisciplinary Healthcare is an international, peer-reviewed open-access journal that aims to represent and publish research in healthcare areas delivered by practitioners of different disciplines. This includes studies and reviews conducted by multidisciplinary teams as well as research which evaluates the results or conduct of such teams or healthcare processes in general. The journal covers a very wide range of areas and welcomes submissions from practitioners at all levels, from all over the world. The manuscript management system is completely online and includes a very quick and fair peer-review system. Visit http://www.dovepress.com/testimonials.php to read real quotes from published authors.

Submit your manuscript here: https://www.dovepress.com/journal-of-inflammation-research-journal 